\title{
Effective Soliton Fusion Process at the Advanced Stage of Supercontinuum Generation in Photonic Crystal Fibers
}

\author{
Rodislav Driben ${ }^{1}$, Nickolai Zhavoronkov ${ }^{2}$ \\ ${ }^{1}$ Department of Physical Electronics, School of Electrical Engineering, Tel Aviv University, Tel Aviv, Israel \\ ${ }^{2}$ Max-Born-Institut for Nonlinear Optics and Short Pulse Spectroscopy, Berlin, Germany \\ Email: zhavoron@mbi-berlin.de, driben@post.tau.ac.il
}

Received July 4, 2012; revised August 5, 2012; accepted August 18, 2012

\begin{abstract}
Soliton fusion is a fascinating and delicate phenomenon that manifests itself in optical fibers in case of interaction between co-propagating solitons with small temporal and wavelengths separation. The mechanism of graduate acceleration of trailing soliton by dispersive waves radiated from the preceding one provides necessary conditions for soliton fusion at the advanced stage of supercontinuum generation in photonic crystal fibers. As a result large intensity robust light structures can propagate over significant distances. In the spectral domain fusion-like processes result in development of a new significant band at the long wavelength side of the spectrum.
\end{abstract}

Keywords: Supercontinuum Generation; Temporal Solitons; Soliton Fusion; Photonic Crystal Fibers

\section{Introduction}

A fascinating process of short laser pulses conversion into light with broad continuous spectra by various nonlinear optical processes, known as the supercontinuum (SC) generation, has attracted a lot of scientific attention over the past few decades. Since the first observation in 1970 in bulk glass [1], numerous investigations were conducted in a wide variety of nonlinear media, including solids $[2,3]$, liquids $[4,5]$, gases [6], and various types of micro $[3,7]$ and nano-structured [8-10] waveguiding media. The supercontinuum does not originate from a specific single phenomenon but rather from a plethora of nonlinear effects, such as self-phase modulation (SPM), high order soliton fission, modulation instability, stimulated Raman scattering (SRS), self-steepening (SS), four wave mixing, which combine to produce an extreme pulse broadening, with the widest and most homogeneous SC spectrum obtained when the pump pulses are launched close to the zero-dispersion wavelength and in the anomalous regime [3]. In the spectral region of long wavelengths where the dispersion is anomalous, solitons play a major role in the SC formation process [11]. They influence pulse spectral broadening at the initial stage of SC formation via a fission process $[3,12]$ and at later stages via their interaction with dispersive waves by means of Kerr and Raman nonlinearities $[11,13,14]$. The aim of the present work is to demonstrate an effective fusion-like processes [15] resultant from multiple interactions both between solitons and dispersive waves and between adjacent solitons at the advanced stage of SC formation. In the spectral domain the process of fusion results in development of a new significant band at the long wavelength side of the spectrum. Since conditions for the effective fusion are very delicate, the event becomes a rare one. Obviously, an enhanced light structure with amplitude maintaining for a long propagation is a very attractive candidate for optical analog of rogue waves [16,17]. The rest of the paper is organized as follows: Theoretical description of the model studied, experimental observations and comparison of experimental data with numerical simulations, main results, conclusions.

\section{Theoretical Model}

To study various physical phenomena involved in the experimentally observed results we use a generalized scalar nonlinear Schroedinger equation (GNLSE) to model the pulse propagation inside the fiber [3]:

$$
\begin{aligned}
\frac{\partial A}{\partial z} & =\sum_{m \geq 2} \frac{i^{m+1} \beta_{m}}{m !} \frac{\partial^{m} A}{\partial T^{m}}-0.5 \alpha A \\
& +i Y\left(1+\frac{i}{w} \frac{\partial}{\partial T}\right)\left[A(z, T) \int_{-\infty}^{T} \mathrm{~d} T^{\prime} R\left(T-T^{\prime}\right)\left|A\left(z, T^{\prime}\right)^{2}\right|\right]
\end{aligned}
$$

where the electric field with amplitude $A$ propagates along the fiber with longitudinal coordinate $z$. Time in a reference frame traveling with the pump light is represented by $T . \beta_{m}$ is the $m$ th order dispersion coefficient at 
the central frequency $\omega_{0}, \alpha$ is the fiber loss. The nonlinear coefficient is $\gamma=n_{2} \omega_{0} /\left(c A_{\text {eff }}\right)$, where $n_{2}=2.4 \times$ $10^{-20} \mathrm{~m}^{2} / \mathrm{W}$ is the nonlinear refractive index of fused-silica glass, and $A_{\text {eff }}$ is the effective modal area of the fiber. The response function is given by

$$
R(T)=\left(1-f_{R}\right) \delta(T)+f_{R} h_{R}(T)
$$

with first and second terms standing for instantaneous and delayed Raman contributions, respectively. $f_{R}=0.18$ is the fraction of Raman contribution to nonlinear polarization, and

$$
h_{R}(T)=\left(\tau_{1}^{2}+\tau_{2}^{2}\right) /\left(\tau_{1} \tau_{2}^{2}\right) \exp \left(-T / \tau_{1}\right) \sin \left(T / \tau_{1}\right)
$$

approximates the Raman response function of silica fiber [18] with $\tau_{1}=12.2 \mathrm{fs}$ and $\tau_{2}=32 \mathrm{fs}$. Equation (1) was solved numerically by using the split-step Fourier method [19]. We consider a typical PCF of core diameter $2.5 \mu \mathrm{m}$ with zero dispersion wavelength $\left(\lambda_{D}\right)=790 \mathrm{~nm}$. At a wavelength of $800 \mathrm{~nm}$, where the input pulse is centered, the nonlinear coefficient is estimated to be $\gamma=$ $80 \mathrm{~W}^{-1} \cdot \mathrm{km}^{-1}$, and the dispersion coefficients up to seventh order are: $\beta_{2}=-2.1 \mathrm{fs}^{2} / \mathrm{mm}, \beta_{3}=69.83 \mathrm{fs}^{3} / \mathrm{mm}, \beta_{4}=$ $-73.25 \mathrm{fs}^{4} / \mathrm{mm}, \beta_{5}=191.95 \mathrm{fs}^{5} / \mathrm{mm}, \beta_{6}=-727.13 \mathrm{fs} / \mathrm{mm}$, $\beta_{7}=1549.4 \mathrm{fs}^{7} / \mathrm{mm}$. Since only a short length of fiber is considered in the simulations, the fiber loss is neglected $(\alpha=0)$. The input pulses are approximated by:

$$
A(0, T)=\sqrt{P_{0}} \operatorname{sech}\left(\frac{T}{T_{0}}\right) \exp \left(\frac{-i c T^{2}}{2 T_{0}^{2}}\right)
$$

where $P_{0}$ is the peak power, $T_{0}$ is related to the FWHM by $T_{\mathrm{FWHM}} \approx 1.763 T_{0}$, and $C=-1.25$ is the parameter representing the initial linear frequency chirp as it appears in the experiment. To study various physical phenomena involved in the experimentally observed results we use a generalized scalar nonlinear Schroedinger equation (GNLSE) to model the pulse propagation inside the fiber [3].

\section{Experimental Setup}

25 -fs pulses centered at $790 \mathrm{~nm}$ with spectral width of 45 $\mathrm{nm}$ FWHM was used in the experiment [20]. The whole experimental setup is shown in Figure 1.

The spatial properties of the output laser beam were found to be excellent, demonstrating symmetrical Gaussian profile with $\mathrm{M}^{2}$ close to 1 . Mirrors introducing negative dispersion of about $-40 \mathrm{fs}^{2} /$ bounce (Layertec $\mathrm{GmbH}$ ) were used to manage the chirp of the pulses. The mirrors were specially designed as a pair with the aim to compensate the oscillation of GVD and to minimize the introduced third order dispersion (TOD). The beam could experience up to 12 bounces within the single NGVD mirror-pair. To increase the amount of the introduced negative dispersion another mirror-pair could be inserted into the beam path. The laser radiation was focused by a microscope objective $(40 \times, \mathrm{NA}=0.65)$ into the photonic crystal fiber (PCF) of $38 \mathrm{~cm}$ length with the diameter of the central rod of $2.5 \mu \mathrm{m}$ and zero dispersion wavelength of $\lambda_{D}=790 \mathrm{~nm}$. The throughput of the PCF exceeds $60 \%$, what was used to calculate peak power insight the PCF. The highly divergent output radiation from the PCF was collimated by an aspheric condensing lens with a 4.6 $\mathrm{mm}$ focal length $(\mathrm{NA}=0.55)$.

The input pulse duration and phase were measured by Spider (APE Berlin). The original laser pulses showed a positive chirp after passing through the focusing optics with the pulseduration extended from initial $25 \mathrm{fs}$ up to 150 fs. This excess of positive dispersion can be to compensated by introducing 24 reflections from NGVD mirror. By inserting or removing one NGVD mirror-pair the pulse duration could be stretched up to $60 \mathrm{fs}$. The chirps in these two cases manifest the opposite signs with additionally introduced GVD of about $\pm 480 \mathrm{fs}^{2}$. The spectra of the radiation after propagation in the PCF were recorded for different values of the input pulse chirp as a function of input pulse power. Comparison of the spectra was performed for equal pulse energies at the level of 20 $\mathrm{dB}$, while the peak power decreased as the chirp grew. Surprisingly, we observed a drastic reduction of the spectral bandwidth for pulses with positive chirp compared to unchirped or negatively chirped pulses. At the same time the spectra for the unchirped and negatively chirped pulses demonstrated very similar dynamics showing development of fundamental solitons at much lower input powers compared to the pulses with the positive chirp. From here on we will analyse the pulses with the same duration of $60 \mathrm{fs}$ and peak intensity, but with the opposite chirps, to clarify only the impact from different sign of chirp on SC dynamics.

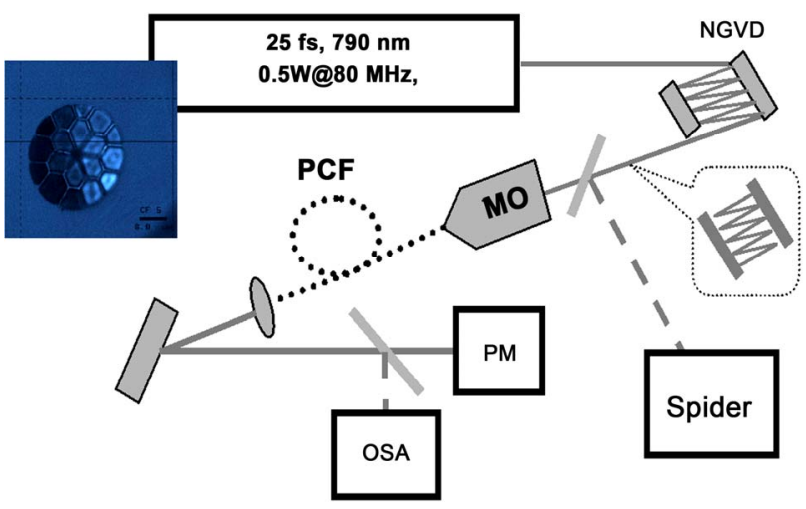

Figure 1. Experimental setup: NGVD, the mirror pair with negative dispersion; PCF, the photonic crystal fiber; MO, the microscopic objective; CL, condensing lens; OSA, the optical spectrum analyzer ANDO AQ-6315A; PM, the power meter. Insert: Cross section of the $2.5-\mu \mathrm{m}$ core diameter PCF with zero dispersion wavelength $790 \mathrm{~nm}$. 


\section{Simple Fusion of Two Fundamental Solitons in the PCF and Fusion of Raman-Shifted Quasi-Solitons at the Advanced Stage of the SC Formation}

To verify the accurateness of numerical simulations vs. the experimental data we modeled pulses dynamics in the PCF for various values of input peak powers. A comparison between experimental data (solid curve) and numerical simulations (dashed curve) for the input peak powers of 2, 10, 50, and $100 \mathrm{~kW}$ is presented in Figure 2 (a). Although the calculated spectra differ slightly from the experimental ones, a good consistency can be recognized in the spectral width and shape. Figure 2(b) demonstrates the spectral bandwidth measured at $20 \mathrm{~dB}$ below maximum in the experiment and calculated by the numerical modeling as a function of increasing input peak power.

Next a simple fusion process between two fundamental non-chirped solitons copropagating in PCF with small initial temporal separation of $0.3 \mathrm{ps}$ was simulated by solving numerically (1) for injected two fundamental solitons at $\lambda_{1}=820 \mathrm{~nm}$ and $\lambda_{2}=755 \mathrm{~nm}$.

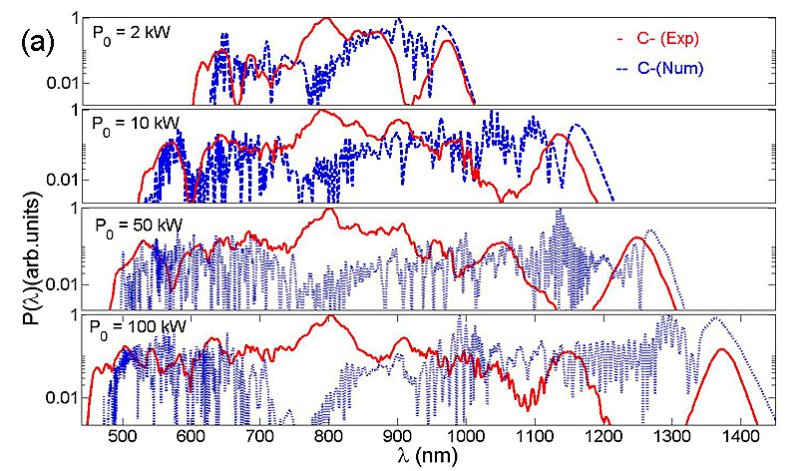

(a)

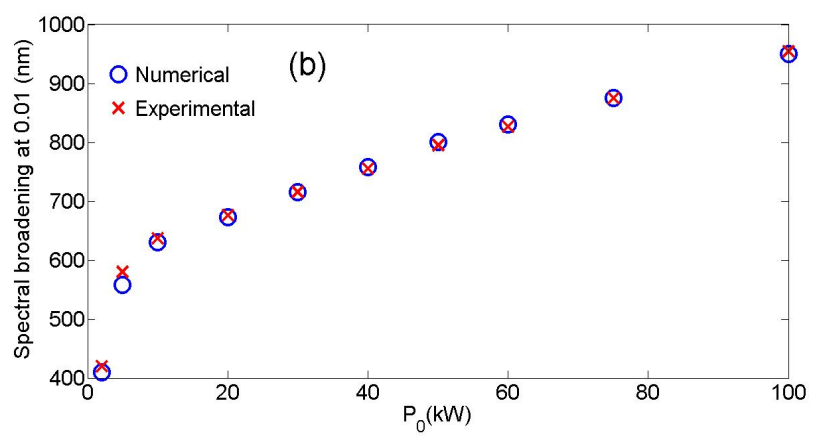

(b)

Figure 2. (Color online) (a) Comparison between spectra observed in the experiment (solid red curve) and calculated numerically (dashed red curve); (b) Spectral broadening at $20 \mathrm{~dB}$ below maximum for negatively prechirped $60 \mathrm{fs}$ pulses with different input power (experimental data + numerics). Underlined are spectral bands resultant from Raman solitons collisions.
Figure 3 clearly demonstrates oblong wave crest resultant from the effective fusion of the two solitons, maintaining for several meters of propagated distance. The initial pulse parameters are: $P_{0}=15.66 \mathrm{~W}, T_{\mathrm{FWHM}}=$ 105 fs. N.B: The maximum height of the crest, as well as it's lengths along the Z-axis are very sensitive on initial conditions such as temporal and frequency separation between the interacting pulses. For example a little larger wavelengths difference would make the crest's peak spike-like typical for soliton's elastic collisions [11]. While slightly smaller wavelength separation would make the crest longer lasting over $\mathrm{Z}$, but with height less pronounced. For the example above delicate initial conditions were specially provided to observe a fusion process in PCF. We demonstrated next, how such conditions could be met in a real-life process of supercontinuum generation resultant from the fission of high intensity higher order soliton injected in PCF. The mechanism of acceleration of trailing soliton by dispersive waves radiated from preceding one was recently demonstrated [21] and further explored in all-optical manipulating of solitons by means of dispersive waves [22].

This mechanism may provide gradual enough decrease of temporal shift between two fission ejected solitons until they meet in fusion-like process rather than in regular quasi-elastic collision [11]. The phenomena we wish to address here do not occur for low input peak powers. Therefore we show the numerically obtained temporal and spectral evolutions of $60 \mathrm{fs}$ (FWHM) input pulses at $100 \mathrm{~kW}$ in Figure 4. The PCF length was $38 \mathrm{~cm}$ as in the experiment.

We now concentrate on details of the interaction between solitons and radiation. Figure 5 shows a zoomed region from Figure 4. The emitted dispersive wave interacts with the next Raman-induced red-shifted soliton [23] and bends the trailing soliton's trajectory so that both solitons approach each other [21]. At the maximum distance shown, which is the entire fiber length, the solitons are about to collide and experience strong energy

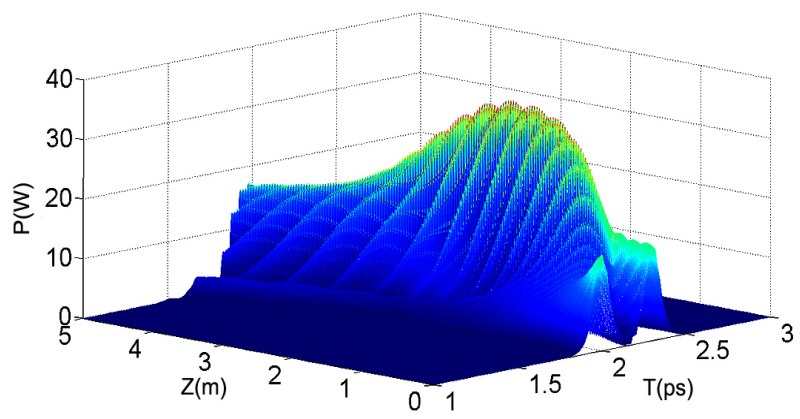

Figure 3. (Color online) Fusion resultant from co-propagation of two fundamental solitons with wavelengths $\lambda_{1}=820$ $\mathrm{nm}$ and $\lambda_{2}=755 \mathrm{~nm}$. Initial pulses separation is $0.3 \mathrm{ps}$ and their widths $T_{\mathrm{FWHM}}=105 \mathrm{fs}$. 

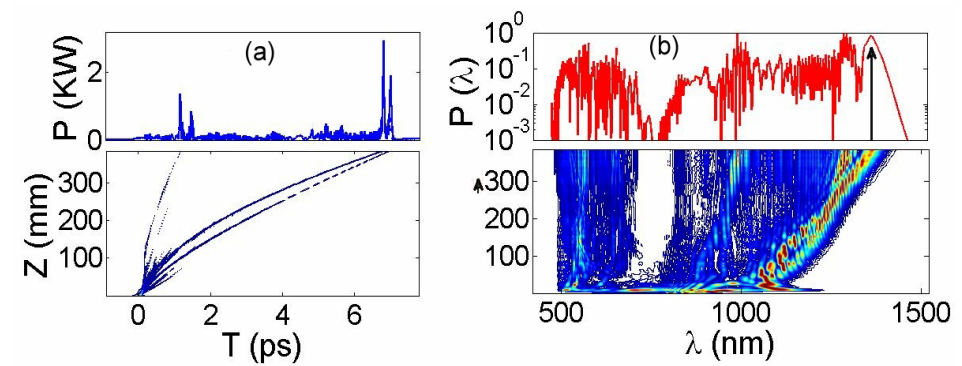

Figure 4. (Color online) Pulse evolution in PCF with input peak power $P_{0}=100 \mathrm{~kW}$ for negatively prechirped $60 \mathrm{fs}$ pulses. (a) Temporal domain; (b) Spectral domain.

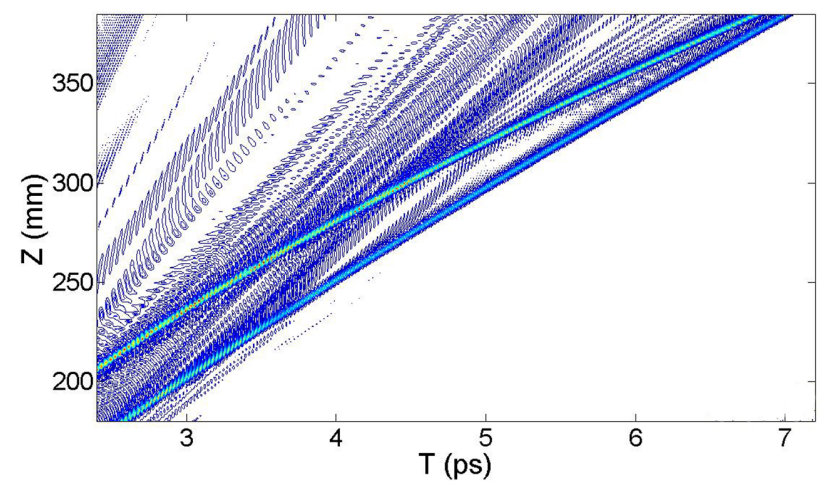

Figure 5. (Color online) Interactions between the radiation and solitary waves with following relative acceleration and collisions between solitons. Zoom of the interaction region for the input pulse of $100 \mathrm{~kW}$.

exchange with the generation of new frequencies. These effects are presented at the long wavelength side of the spectrum extending the spectrum from $480 \mathrm{~nm}$ up to $1420 \mathrm{~nm}$ measured at $20 \mathrm{~dB}$ below maximum in Figure 4(b). In [21] we have proven that indeed the emitted radiation is responsible for the trajectory bending. We performed an additional simulation very similar to the one of Figure 4, but with artificially eliminating most of the radiation in the temporal window between the two solitons and observed very little relative acceleration. The interaction of Raman red-shifted solitons with dispersive waves is extremely sensitive to the initial parameters. Additional simulations were performed to determine the influence of the chirp parameter and higher-order dispersion of the PCF. In order to optimize fusion between solitons we performed multiple simulations with variation of injected peak power, chirp parameters and pulse widths along with random small noise realization in input.

Figures 6(a) and (b) demonstrates an example of an effective soliton fusion at $C=-1.16$ when the colliding solitons combine into one giant solitary wave which keeps propagating at large group velocity. In the process a strong spectral band is generated, which extends the SC spectrum beyond $1500 \mathrm{~nm}$.

Another extremely important parameter of the system
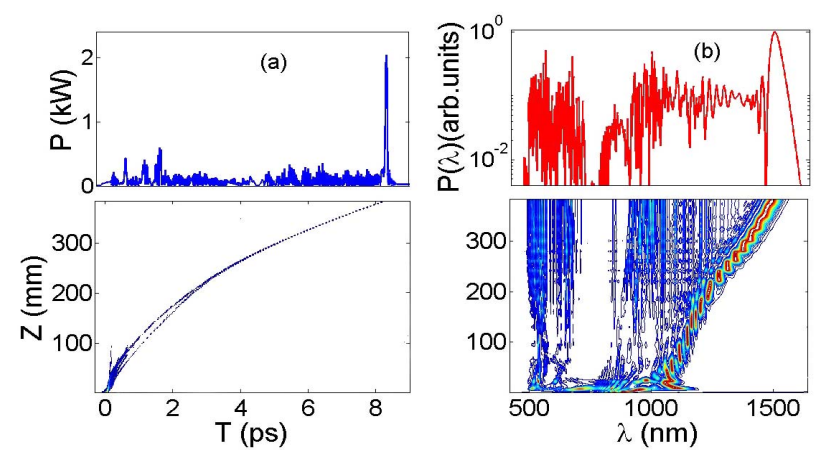

Figure 6. (Color online) Pulse evolution in PCF with input peak power $P_{0}=100 \mathrm{~kW}$, with $C=-1.16$. (a) Temporal domain; (b) Spectral domain.

is the dispersion profile of the PCF [4]. We performed an additional simulation excluding higher than $3^{\text {rd }}$ order dispersion and observed that the solitons repelled each other rather than attracted [21]. It is also worth to point out, that fusion events were observed when the light is injected deeper in the anomalous dispersion region $(\lambda>$ $790 \mathrm{~nm}$ ). In this region solitons ejection from fission process are more frequent with shorter temporal delay between neighboring solitons, thus enabling an accelerated soliton to catch up with the preceding one.

Fusion of solitons result in large intensity robust light structures propagates over significant distance. The effect can further be enhanced by multiple fusion-like events [17]. In presence of small random noise the delicate condition for the fusion-like interaction between solitons becomes a statistical matter. Thus oblong-shaped giant accelerated waves become best resembling candidates for an optical analog of oceanic rogue waves.

\section{Conclusion}

In conclusion, we have studied experimentally and numerically dynamics of high-power ultrashort negatively prechirped pulses in PCFs resulting in broad supercontinuum generation. Effective solitons fusion process was demonstrated at the advanced stage of the supercontinuum formation. The soliton fusion process requires very delicate conditions for colliding solitons, such as 
small temporal and frequency separation. It was demonstrated, that acceleration of trailing soliton by dispersive waves radiated from preceding one may cause to graduate attraction between the solitons with a resultant fusion. Fusion-like processes are more likely to occur when the high intensity light is injected deeper in the anomalous dispersion region, with frequently ejected solitons at the initial fission process of the input higher order soliton.

\section{REFERENCES}

[1] R. R. Alfano and S. L. Shapiro, "Observation of SelfPhase Modulation and Small-Scale Filaments in Crystals and Glasses," Physical Review Letter, Vol. 24, No. 11, 1970, pp. 592-594. doi:10.1103/PhysRevLett.24.592

[2] R. R. Alfano, "The Supercontinuum Laser Source: Fundamentals with Updated References," 2nd Edition, Springer, New York, 2006. doi:10.1007/b106776

[3] J. M. Dudley, G. Gentry and S. Coen, "Supercontinuum Generation in Photonic Crystal Fiber," Review of Modern Physics, Vol. 78, No. 4, 2006, pp. 1135-1184. doi:10.1103/RevModPhys.78.1135

[4] P. Ho, Q. X. Li, T. Jimbo, Y. L. Ku and R. R. Alfano, "Supercontinuum Pulse Generation and Propagation in a Liquid Carbontetrachloride," Applied Optics, Vol. 26, No. 14, 1987, pp. 2700-2702. doi:10.1364/AO.26.002700

[5] R. Driben, A. Husakou and J. Herrmann, "Supercontinuum Generation in Aqueous Colloids Containing Silver Nanoparticles," Optics Letters, Vol. 34, No. 14, 2009, pp. 2132-2134. doi:10.1364/OL.34.002132

[6] P. B. Corkum, C. Rolland and T. Srinivasan-Rao, "Supercontinuum Generation in Gases," Physical Review Letter, Vol. 57, No. 18, 1986, pp. 2268-2271. doi:10.1103/PhysRevLett.57.2268

[7] J. C. Travers, M. H. Frosz and J. M. Dudley, "3-Nonlinear Fibre Optics Overview," In: J. M. Dudley and J. R. Taylor, Eds., Supercontinuum Generation in Optical Fibers, Cambridge University Press, Cambridge, 2010, pp. 32-51. doi:10.1017/CBO9780511750465.004

[8] M. Foster, A. Gaeta and R. Trebino, "Soliton-Effect Compression of Supercontinuum to Few-Cycle Durations in Photonic Nanowires," Optics Express, Vol. 13, No. 18, 2005, pp. 6848-6855.

[9] R. Driben, A. Husakou and J. Herrmann, "Supercontinuum Generation in Aqueous Colloids Containing Silver Nanoparticles," Optics Letters, Vol. 34, No. 14, 2009, pp. 2132-2134. doi:10.1364/OL.34.002132

[10] N. Zhavoronkov, R. Driben, B. A. Bregadiolli, M. Nalin and B. A. Malomed, "Observation of Asymmetrical Spectrum Broadening Induced by Silver Nanoparticles in a Heavy-Metal Oxide Glass," Europhysics Letters (EPL), Vol. 94, No. 3, 2011, p. 37011.

[11] D. V. Skryabin and A. V. Gorbach, "Looking at a Soliton through the Prism of Optical Supercontinuum," Review of Modern Physics, Vol. 82, No. 2, 2011, pp. 1287-1299. doi:10.1103/RevModPhys.82.1287

[12] J. Herrmann, U. Griebner, N. Zhavoronkov, A. Husakou, D. Nickel, J. C. Knight, W. J. Wadsworth, P. St. J. Russell and G. Korn, "Experimental Evidence for Supercontinuum Generation by Fission of Higher-Order Solitons in Photonic Fibers," Physical Review Letter, Vol. 88, No. 17, 2002, p. 173901. doi:10.1103/PhysRevLett.88.173901

[13] A. Efimov, A. V. Yulin, D. V. Skryabin, J. C. Knight, N. Joly, F. G. Omenetto, A. J. Taylor and P. Russell, "Interaction of an Optical Soliton with a Dispersive Wave," Physical Review Letter, Vol. 95, No. 21, 2005, Article ID: 213902. doi:10.1103/PhysRevLett.95.213902

[14] F. Luan, D. V. Skryabin, A. V. Yulin and J. C. Knight, "Energy Exchange between Colliding Solitons in Photonic Crystal Fibers," Optics Express, Vol. 14, No. 21, 2006, pp. 9844-9853. doi:10.1364/OE.14.009844

[15] C. Rotschild, B. Alfassi, M. Segev and O. Cohen, "LongRange Interactions between Optical Solitons," Nature Physics, Vol. 2, No. 11, 2006, pp. 769-774. doi: $10.1038 /$ nphys 445

[16] D. R. Solli, C. Ropers, P. Koonath and B. Jalali, "Optical Rogue Waves," Nature, Vol. 450, No. 7172. 2007, pp. 1054-1058. doi:10.1038/nature06402

[17] R. Driben and I. Babushkin, "Accelerated Rogue Waves Generated by Soliton Fusion at the Advanced Stage of Supercontinuum Formation in Photonic Crystal Fibers," Workshop on Laser-Matter Interaction, Porquerolles, 25-29 June 2012, p. 9.

[18] K. J. Blow and D. Wood, "Theoretical Description of Transient Stimulated Raman Scattering in Optical Fibers," IEEE Journal of Quantum Electron, Vol. 25, No. 12, 1989, pp. 2665-2673. doi:10.1109/3.40655

[19] G. P. Agrawal, "Nonlinear Fiber Optics," 4th Edition, Academic Press, Waltham, 2007.

[20] R. Driben and N. Zhavoronkov, "Supercontinuum Spectrum Control in Microstructure Fibers by Initial Chirp Management," Optics Express, Vol. 18, No. 16, 2010, pp. 16733-16738. doi:10.1364/OE.18.016733

[21] R. Driben, F. Mitschke and N. Zhavoronkov, "Cascaded Interactions between Raman Induced Solitons and Dispersive Waves in Photonic Crystal Fibers at the Advanced stage of Supercontinuum Generation," Optics Express, Vol. 18, No. 25, 2010, pp. 25993-25998. doi:10.1364/OE.18.025993

[22] A. Demircan, S. Amiranashvili and G. Steinmeyer, "Controlling Light by Light with an Optical Event Horizon," Physical Review Letter, Vol. 106, No. 16, 2011, Article ID: 163901. doi:10.1103/PhysRevLett.106.163901

[23] F. M. Mitschke and L. F. Mollenauer, "Discovery of the Soliton Self-Frequency Shift," Optics Letters, Vol. 11, No. 10, 1986, pp. 659-661. doi:10.1364/OL.11.000659 